\title{
VORTIOXETINE-INDUCED HYPOMANIA: A CASE REPORT
}

\author{
Emrah Songur \\ Republic of Turkey Ministry of Health, Health Sciences University, Ankara Training and Research Hospital, \\ Ankara, Turkey
}

received: 17.1.2021;

revised: 27.2.2021;

accepted: 4.3 .2021

\section{INTRODUCTION}

Vortioxetine is a new generation antidepressant that has $5-\mathrm{HT}_{3}, 5-\mathrm{HT}_{1 \mathrm{D}}, 5-\mathrm{HT}_{7}$ antagonist, 5- $\mathrm{HT}_{1 \mathrm{~B}}$ partial agonsite and $5-\mathrm{HT}_{1 \mathrm{~A}}$ receptor agonist features (Sanchez et al. 2015). The antagonist effect on 5- $\mathrm{HT}_{3}$ receptors causes nausea and vomiting, the most common side effect of vortioxetine (D'Agostino et al. 2015). In animal studies, it blocks $5-\mathrm{HT}_{3}$ receptors, causing an increase in serotonin, dopamine, norepinephrine, acetylcholine, and histamine (Sanchez et al. 2015).

It is known that manic or hypomanic switch may occur due to antidepressant treatment. Treatments that increase dopamine and norepinephrine levels can cause a manic shift (Allain et al. 2017). The features that increase the risk of manic or hypomanic switch due to antidepressants are female sex, young age, family history of psychiatric disorders, atypical features and three or more lifetime episode of depression (Pirdogan Aydin et al. 2019).

The aim of this case report is to present a patient who presented to psychiatry with depressive complaints for the first time, and a hypomanic shift that occurred two months after the start of vortioxetine with the diagnosis of major depressive disorder.

\section{CASE REPORT}

A 35 year-old woman applied to our clinic because of more than 10 years of unhappiness, not enjoying life and crying spells. She has not applied to psychiatry before. She was started on vortioxetine $10 \mathrm{mg} /$ day with the diagnosis of depression. Then she came to the control with a delay of two months. In her second application, she said that he benefited from vortioxetine which was started earlier, the complaint of unhappiness decreased, but she did not continue drug treatment after a month. Insomnia complaints were accompanied by old complaints. Since she previously benefited from vortioxetine, vortioxetine was started again at $10 \mathrm{mg} /$ day. Trazodone was added to $50 \mathrm{mg} /$ day treatment for insomnia. After two months of treatment, she applied to our clinic, saying that her need for sleep has decreased, she complained about the acceleration of her speech, and that her desire to deal with many projects at work began to increase for last one week. She was still using vortioxetine and had used the trazodone for insomnia for one or two times. Recently she has used no other drugs. There were no pathologic findings that could cause hypomania in the laboratory tests. She has no other known physical disease. Her uncle had a history of substance abuse and died as a result of suicide. There was no manic or hypomanic period before. Vortioxetine treatment was discontinued considering that the patient had a hypomanic shift due to vortioxetine. Young Mania Rating Scale score was 17. She was started on quetiapine $50 \mathrm{mg} /$ day. Since she had concerns about excessive daytime sedation at work as a side effect, quetiapine dose was kept low and a control visit was recommended for one week later. In the follow-up visit, the Young Mania Rating Scale score was 13 . Her speech was slower, her desire to deal with many projects decreased. Quetiapine dose was increased to $100 \mathrm{mg} /$ day. At the control 2 weeks later, the Young Mania Rating Scale score was 2. Quetiapine treatment was continued as $100 \mathrm{mg} /$ day.

\section{DISCUSSION}

In this case report we present a young woman with major depressive disorder who has a hypomanic switch after two month vortioxetine treatment. It was thought that there might be a trazodon induced hypomania since the patient was a two-month period when he did not experience hypomanic symptoms while using vortioxetine alone and although trazodone is thought to be safe at low doses to promote sleep in bipolar patients, in the literature trazodone added to the treatment for insomnia complaints has been reported to cause manic and hypomanic shifts ( $\mathrm{Hu}$ et al. 2017). However, since the patient used trazodone for insomnia for one or two nights two months ago, and then discontinued the drug, as it was evaluated that the hypomanic shift may be due to vortioxetine. Although a meta-analysis on the use of vortioxetine in depression patients known to have no bipolar disorder history has been reported to have no risk of manic shift as a side effect (Berhan \& Barker 2014) and in a study on the safety and tolerability of vortioxetine, only one patient in 3018 patients showed symptoms of 
hypomania, and vortioxetine was not different from placebo in terms of hostility/aggression-related side effects (Baldwin et al. 2016). In the previous literature there are three manic (D'Andrea et al. 2019, Maud 2016, Sobreira et al. 2017) and two hypomanic (Baldwin et al. 2016, Pirdogan Aydin et al. 2019) cases that developed after vortioxetine use. Three of these cases (D'Andrea et al. 2019; Pirdogan Aydin et al. 2019, Sobreira et al. 2017) patients had no known bipolar disorder history, while one case (Maud 2016) had a prior diagnosis of bipolar disorder. Our case is the sixth case of mood shift associated with vortioxetine. Our patient applied to psychiatry for the first time despite having depressive complaints for more than 10 years and had not been diagnosed with bipolar before. There were no known relatives who had bipolar disorder in the family, but his uncle had a history of substance abuse and completed suicide. Being young and female were risk factors for manic / hypomanic switch due to antidepressants for our case.

\section{CONCLUSION}

Vortioxetine is a new antidepressant with its multinodal feature, which has effects on multiple neurotransmitter systems, including dopamine and norepinephrin (D'Agostino et al. 2015). As in this case, the presence of hypomanic and manic switches due to vortioxetine in the literature suggests that there is a risk of manic or hypomanic switch for vortixetine as in other antidepressant treatments. When starting vortioxetine treatment, it may be useful to question whether there is bipolar disorder in the past psychiatric history and family history, to reduce the risk of possible hypomanic or manic shift.

\section{Acknowledgments: None.}

Conflict of interest: None to declare.

\section{References}

1. Allain N, Leven C, Falissard B, Allain JS, Batail JM, Polard E et al.: Manic switches induced by antidepressants: an umbrella review comparing randomized controlled trials and observational studies. Acta Psychiatr Scand 2017; 135:106-116. https://doi.org/10.1111/acps. 12672

2. Baldwin DS, Chrones L, Florea I, Nielsen R, Nomikos GG, Palo $W$ et al.: The safety and tolerability of vortioxetine: Analysis of data from randomized placebo-controlled trials and open-label extension studies. J Psychopharmacol 2016; 30:242-252. https://doi.org/10.1177/0269881116628440

3. Berhan $A \&$ Barker A: Vortioxetine in the treatment of adult patients with major depressive disorder: a metaanalysis of randomized double-blind controlled trials. BMC Psychiatry 2014; 14:276. https://doi.org/10.1186/s12888-014-0276-x

4. D'Agostino A, English CD, Rey JA: Vortioxetine (brintellix): a new serotonergic antidepressant. $P T$ 2015; 40:36-40. https://www.ncbi.nlm.nih.gov/pubmed/25628505

5. D'Andrea G, De Ronchi D, Giaccotto L, Albert U: Vortioxetine treatment-emergent mania in the elderly: a case report. Australas Psychiatry 2019; 27:413. https://doi.org/10.1177/1039856219839471

6. Ни J, Hu L, Zheng $H, H u S, X u Y:$ Fan the flame: trazodone-induced mania in a unipolar depressed patient with stable sertraline treatment. Neuropsychiatric Disease and Treatment 2017; 13:2251-2254

7. Maud C: Vortioxetine in bipolar depression induces a mixed/manic switch. Australas Psychiatry 2016; 24:206-207. https://doi.org/10.1177/1039856215614986

8. Pirdogan Aydin E, Dalkiran M, Ozer OA, Karamustafalioglu KO: Hypomanic switch during vortioxetine treatment: a case report. Psychiatry and Clinical Psychopharmacology 2019; 29:114-116. https://doi.org/10.1080/24750573.2018.1435602

9. Sanchez C, Asin KE, Artigas F: Vortioxetine, a novel antidepressant with multimodal activity: review of preclinical and clinical data. Pharmacol Ther 2015; 145:43-57. https://doi.org/10.1016/j.pharmthera.2014.07.001

10. Sobreira G, Oliveira J, Brissos S.: Vortioxetine-induced manic mood switch in patient with previously unknown bipolar disorder. Braz J Psychiatry 2017; 39:86. https://doi.org/10.1590/1516-4446-2016-2113

\author{
Correspondence: \\ Emrah Songur, MD \\ Republic of Turkey Ministry of Health, Health Sciences University, \\ Ankara Training and Research Hospital \\ Serhat Mah. 1325. Sok Yenimahalle, Ankara Turkey \\ E-mail:emrahsongur@gmail.com
}

